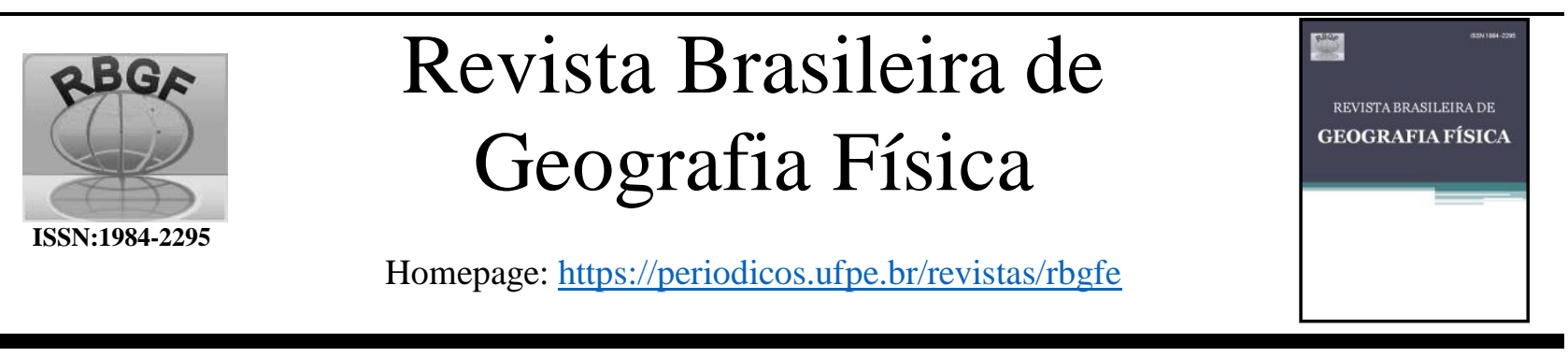

\title{
Análise espacial da tuberculose em Santa Catarina correlacionando com determinantes sociais e de saúde
}

\begin{abstract}
Sérgio Adam Mendonça ${ }^{1}$, Selma Cristina Franco ${ }^{2}$, Celso Voos Vieira ${ }^{3}$, Rosana Leal do Prado ${ }^{4}$
${ }^{1}$ Professor de Clínica Médica Fundação Universidade Regional de Blumenau. E-mail: sergioadam @ terra.com.br. ${ }^{2}$ Selma Cristina Franco. Professora da Universidade da Região de Joinville - Univille. E-mail: scfranco@ terra.com.br. ${ }^{3}$ Celso Voos Vieira. Professor nos cursos de Engenharia Ambiental e Sanitária, Biologia e Biologia Marinha, Engenharia Civil e Arquitetura da Universidade da Região de Joinville - Univille. E-mail: xcelsox@ gmail.com. ${ }^{4}$ Rosana Leal do Prado. Professora do Departamento de Saúde Coletiva em Odontologia da Universidade do Oeste Paulista/Unoeste e professora visitante da Fundação Universidade Regional de Blumenau. E-mail: rosanahb@ gmail.com.
\end{abstract}

Artigo recebido em 03/07/2020 e aceito em 17/11/2020

\section{R E S U M O}

A distribuição espacial da tuberculose e sua relação com marcadores demográficos, ambientais e socioeconômicos tem gerado informações relevantes para aprimorar a vigilância epidemiológica e a avaliação de serviços de saúde. O objetivo deste trabalho é determinar a distribuição espacial da tuberculose no Estado de Santa Catarina entre 2005 e 2015 , correlacionando com indicadores socioeconômicos, demográficos e de saúde. Realizou-se um estudo ecológico cuja análise espacial utilizou a técnica de polígonos. $\mathrm{Na}$ análise exploratória foram utilizadas as taxas brutas de incidência e taxas suavizadas pelo método bayesiano empírico. A autocorrelação espacial das taxas de incidência brutas e suavizadas foi determinada pelo índice Global de Moran e clusters pelo índice Local de Moran. Realizou-se uma modelagem com 20 indicadores utilizando a variável dependente "incidência de tuberculose" e regressão multivariada. A distribuição da tuberculose apresentou correlação espacial positiva com maior concentração na região nordeste do estado e, também, na faixa litorânea $(\mathrm{p}=0,001)$. No melhor modelo houve correlação inversa entre a incidência de tuberculose e o índice de Gini ( $\mathrm{p}<0,001)$, e correlação direta com a taxa de desocupação de 18 anos ou mais $(\mathrm{P}<0,001)$, percentual de pessoas de 15 a 24 anos vulneráveis $(p=0,011)$, domicílios com mais de duas pessoas por dormitório $(\mathrm{p}=0,011)$, aumento da população $(p=0,003)$ e incidência de HIV $(p<0,001)$. Conclui-se que em Santa Catarina há autocorrelação espacial da tuberculose com concentração nos conglomerados urbanos das maiores cidades, nas quais o padrão socioeconômico é mais elevado com aumento populacional recente e receptoras de fluxo migratório.

Palavras-chave: Tuberculose. Georreferenciamento. Análise espacial. Indicadores socioeconômicos, demográficos e de saúde.

\section{Spatial analysis of tuberculosis in Santa Catarina correlating with social and health determinants}

\begin{abstract}
A B S T R A C T
The spatial distribution of tuberculosis and its relationship with demographic, environmental and socioeconomic markers has generated relevant information to improve epidemiological surveillance and the evaluation of health services. The objective of this work is to determine the spatial distribution of tuberculosis in the State of Santa Catarina between 2005 and 2015, correlating with socioeconomic, demographic and health indicators. An ecological study was carried out whose spatial analysis used the polygon technique. In the exploratory analysis, crude incidence rates and rates smoothed by the empirical Bayesian method were used. The spatial autocorrelation of crude and smoothed incidence rates was determined by the Global Moran index and clusters by the Local Moran index. A modeling was carried out with 20 indicators using the dependent variable "incidence of tuberculosis" and multivariate regression. The distribution of tuberculosis showed a positive spatial correlation with greater concentration in the northeastern region of the state and also in the coastal strip $(\mathrm{p}=0.001)$. In the best model, there was an inverse correlation between the incidence of tuberculosis and the Gini index ( $\mathrm{p}<0.001)$, and a direct correlation with the unemployment rate of 18 years or older $(\mathrm{P}<0.001)$, the percentage of people aged 15 to 24 who are vulnerable $(\mathrm{p}=0.011)$, households with more than two people per bedroom $(\mathrm{p}=0.011)$, population increase $(\mathrm{p}=0.003)$ and HIV incidence $(\mathrm{p}<0.001)$. It is concluded that in Santa Catarina there is spatial autocorrelation of tuberculosis with concentration in the urban conglomerates of the largest cities, in which the socioeconomic standard is higher with a recent population increase and receptors of migratory flow.
\end{abstract}

Keywords: Tuberculosis. Georeferencing. Spatial analysis. Socioeconomic, demographic and health indicators. 


\section{Introdução}

A tuberculose é uma doença infectocontagiosa, de distribuição universal, mas com um fardo maior nos países de baixa renda (World Health Organization, 2015, 2016). É uma doença de difícil erradicação, apesar de possuir tratamento eficaz, pela multiplicidade de fatores que interferem em sua prevalência. É um grave problema social, por ser uma doença ligada a populações carentes e excluídas das políticas sociais (Bergonzoli et al., 2016).

A transcendência de seu estudo se apoia no imenso impacto social, uma vez que a doença acomete preferentemente as populações e segmentos da comunidade mais vulneráveis. Para muitos países e regiões, incluindo o Brasil, o controle de tuberculose é ainda uma realidade distante (World Health Organization, 2018).

O agente etiológico é o Mycobacterium tuberculosis, que é transmitido do doente, especialmente da forma pulmonar, para o indivíduo suscetível, através de aerossóis produzidos pela fala, tosse ou espirro (Dheda et al., 2016).

A Organização Mundial da Saúde - OMS relatou em 2018 a ocorrência de 10 milhões de casos novos de tuberculose no mundo, com 1,2 milhões de mortes entre pessoas HIV negativos e um adicional de 251.000 óbitos em portadores do HIV, com uma taxa de coinfecção TB-HIV de $8,6 \%$. Foram acometidos pacientes de todas as faixas etárias, mas $89 \%$ foram adultos maiores de 15 anos (World Health Organization, 2018).

O Brasil ocupa a $20^{a}$ posição entre os países quanto à carga da doença e $19^{\mathrm{a}}$ posição segundo a taxa de coinfecção TB-HIV (Ministério da Saúde, 2017).

Dados mais recentes do Ministério da Saúde informam que foram registrados 72.788 casos novos de TB em 2018, e em 2017, foram registrados 4.534 óbitos pela doença, o que equivale a um coeficiente de mortalidade de 2,2 óbitos/100 mil habitantes (Ministério da Saúde, 2019 (Brasil,2019).

A tuberculose tem raízes profunas na pobreza, devido à propagação facilitada pela precariedade de condições de vida, dificuldade de acesso ao sistema de saúde e baixa adesão ao tratamento. Esta situação tende a perenizar no tempo e no espaço, pelas características próprias da doença e de seus determinantes socioeconômicos (San Pedro et al., 2017, de Souza et al., 2020).

Entretanto, ao se comparar os elevados indicadores sociais e de desenvolvimento verificados no Estado de Santa Catarina com a evolução dos indicadores epidemiológicos da tuberculose, observa-se um paradoxo. Enquanto no Brasil vem ocorrendo uma diminuição das taxas de incidência da doença nas últimas décadas, no estado de Santa Catarina, não tem sido observada a mesma tendência de queda (Secretaria Estadual de Saúde de Santa Catarina, 2017, Ministério da Saúde, 2016). A nível mundial a queda na taxa de incidência também tem sido observada e, segundo dados da Organização Mundial de Saúde, a taxa de tuberculose vem declinando globalmente no período entre 2000 a 2018, sendo que a média da redução foi de $1,6 \%$ ao ano (World Health Organization, 2019).

Quando se leva em conta a organização do sistema de saúde no que tange ao atendimento à tuberculose, estudo de análise espacial da qualidade da atenção básica em saúde no Brasil, mostrou que o Estado de Santa Catarina concentra a maior proporção de municípios entre os mais bem avaliados (Abreu et al., 2018). No âmbito do Sistema único de Saúde (SUS) cabe à atenção básica o papel principal nas ações de controle da tuberculose. Trata-se portanto de um estado onde a atenção à saúde está estruturada com qualidade.

Permanece assim essa lacuna na compreensão dos fatores que vem determinando a manutenção das taxas de incidência da tuberculose em Santa Catarina, diversamente do que se poderia esperar.

Uma abordagem que vem ganhando relevância em estudos epidemiológicos sobre a ocorrência da tuberculose é sobre a distribuição espacial das doenças, em especial da tuberculose, e sua relação com marcadores demográficos, ambientais e socioeconômicos, gerando informações relevantes para os campos da vigilância epidemiológica, avaliação de serviços de saúde, urbanização e influência desta nas condições na saúde (Gehlen et al., 2019, Kolifarhood et al., 2015).

Os sistemas de informação geográfica (SIG) vêm sendo cada vez mais utilizados para o planejamento, gestão, monitoramento e caracterização de espaços geográficos em saúde pública (Guimarães, 2016, L. M. M. De Lima et al., 2019). Tais ferramentas e tecnologias emergentes, permitem gerar mapas que possibilitam conhecer mais detalhadamente as condições de saúde da população, observar sua distribuição espacial, identificar situações de risco pela integração de dados demográficos, socioeconômicos e ambientais (Bonifácio et al., 2019, Schootman et al., 2016). A utilização de dados espaciais em análises epidemiológicas geram informações relevantes para a coordenação de políticas de 
saúde e vigilância em saúde pública (Lee et al., 2016).

Em Santa Catarina, até presente momento, há uma carência deste tipo de estudos, especialmente em relação à tuberculose, por isso, $\mathrm{o}$ objetivo deste trabalho é determinar a distribuição espacial e o grau de autocorrelação espacial da tuberculose no Estado de Santa Catarina entre 2005 e 2015, correlacionando com indicadores socioeconômicos, demográficos e de saúde.

\section{Material e Métodos}

Trata-se de um estudo ecológico da distribuição espacial da tuberculose no estado de Santa Catarina, no período de 2005 a 2015. Para a análise espacial foi utilizada a técnica de área, cujos polígonos foram os 295 municípios do estado. Inicialmente foi feita uma análise exploratória dos dados seguida da modelagem espacial (Brasil, 2007).

Foi considerada variável dependente a média da incidência de tuberculose no período (Ministério da Sasúde, 2018), que da mesma forma que as variáveis independentes, foram obtidas de fontes oficiais para cada município.

Os níveis de corte definidos para a taxa de incidência por 100 mil habitantes foram: de 0 a 1 muito baixa, de 1 a 10 baixa, de 10 a 26,9 elevada abaixo da média do estado, de 26,9 a 37,5 elevada, entre a média do estado e a média nacional, de 35,7 a 53,8 elevada, maior que a média nacional até duas vezes a média do estado e maior que 53,8 muito elevada (Clancy et al., 1991). Os cortes acima de 10 por 100 mil, foram comparados com a média do estado e com a média nacional e com duas vezes a média do estado.

Foram considerados indicadores demográficos:, estimativa do aumento proporcional da população em relação ao censo de 2000 e sua projeção em 2016, densidade demográfica, proporção da população residente na área urbana, taxa de envelhecimento, Razão de dependência (Programa das Nações Unidas para o Desenvolvimento, Instituto de Pesquisa Econômica Aplicada, 2012; Ministério da Saúde, 2018). Como variáveis socioeconômicas consideraram-se: índice de Gini, proporção de vulneráveis à pobreza, proporção da população em domicílios com abastecimento de água e esgotamento sanitário inadequados, proporção de pessoas em domicílios com paredes inadequadas, proporção da população vivendo em domicílios com densidade superior a 2 pessoas por dormitório, proporção de pessoas de 15 a 24 anos que não estudam nem trabalham e são vulneráveis à pobreza, proporção de mães chefes de família sem ensino fundamental completo e com filhos menores de 15 anos, proporção de pessoas com 18 anos ou mais sem o fundamental completo e em ocupação informal, índice de Desenvolvimento Humano municipal, Produto Interno Bruto por município, taxa de desocupação 18 anos ou mais (Programa das Nações Unidas para o Desenvolvimento, Instituto de Pesquisa Econômica Aplicada, 2012; Ministério da Saúde, 2018) e os indicadores de saúde, taxa de mortalidade infantil, incidência média de tuberculose no período, incidência média de HIV no período, média do percentual de cobertura da Estratégia de Saúde de Família no período (Programa das Nações Unidas para o Desenvolvimento, Instituto de Pesquisa Econômica Aplicada, 2012; Ministério da Saúde, 2016; Ministério da Saúde, 2018).

Foi utilizada análise exploratória através da visualização dos dados por intermédio de mapas temáticos e identificação dos padrões de dependência espacial da variável dependente do estudo, que é a incidência de tuberculose. Neste trabalho foi utilizado como indicador global o índice de Moran Global e indicador local, índice de Moran Local (Luzardo et al., 2017).

Em seguida, os dados foram submetidos à modelagem, ou seja, escolha do modelo que melhor explicaria a associação das variáveis ( Brasil, 2007).

Foi realizada análise descritiva dos dados, de modo a se obter a amplitude, média, mediana, desvio padrão e desvio interquartílico das variáveis. Para se adequar aos pressupostos deste tipo de análise, as vinte medidas descritivas, que incluem a variável dependente, taxa média de incidência de tuberculose nos municípios de Santa Catarina no período de 2005 a 2015, foram submetidas a um Teste de Normalidade Kolmogorov Smirnov para verificação de normalidade.

As variáveis independentes com distribuição paramétrica foram submetidas ao teste de correlação de Pearson e as não paramétricas ao teste de Spearman com a taxa média de incidência de tuberculose no período. A variáveis que apresentaram correlação muito forte entre si foram retiradas do modelo para corrigir questões relacionadas à multicolinearidade (McClelland et al., 2016).

Para determinar as variáveis independentes a serem utilizadas nos modelos de regressão, uma vez constatada a autocorrelação espacial, foi considerada uma significância estatística de $05 \%$ e $\mathrm{R}<0,7$, onde R é o coeficiente de correlação. Para 
o índice de Gini foi considerado $0,8 \%$, conforme as recomendações de Hosmer et al. (2013).

Visando diminuir a instabilidade das taxas brutas não associadas ao risco de ocorrência do evento, foi aplicado o alisamento das taxas de incidência por meio do método Bayesiano Empírico local (Magalhães e Medronho 2017). Verificando-se que a distribuição dos resíduos não possuía distribuição normal, empregou-se a transformação dos dados da variável dependente por meio de logaritmo neperiano (Brasil, 2007).

A presença de aglomerados foi determinada de acordo com o índice de Moran Global. Contudo, para examinar padrões numa escala de maior detalhe e verificar se a hipótese de estacionariedade do processo permaneceria localmente, utilizou-se índice de Moran Local, Local Indicators of Spatial Autocorrelation (LISA) (Anselin, 1995). Dada sua característica de indicador local, calcula valor específico de correlação para cada área, permitindo assim, a identificação de clusters de áreas e outliers (Jerrett et al., 2010). Onde os alglomerados ou clusteres são classificados como alto-alto se apresentarem municípios com incidência elevada, próximos à municípios também de incidência elevada, baixobaixo se municípios com incidência baixa estiverem próximos à municípios com incidência baixa, e alto-baixo se município com taxa elevada forem contíguos à municípios com taxa de incidência baixa e alto- baixo o inverso, município com alta incidência contíguo a municípios com baixa incidência.

Foi utilizada a técnica de regressão linear multivariada Ordinary Least Squares Estimation (OLS) com método backward, buscando por variáveis explicativas que melhor descrevessem a ocorrência da doença com nível de confiança de 95\% (Righetto e Tachibana, 2015).

Embora o OLS não seja o método ideal de análise de dados espaciais é sempre o procedimento inicial para as análises de regressão espacial com a finalidade de verificar se uma mudança ocorrida em uma ou mais variáveis independentes modifica a variável dependente (Garcia e Silva, 2016).
Com objetivo de agregar os efeitos espaciais, foram utilizados outros dois modelos, os quais incorporam à regressão tradicional um parâmetro espacial. Dessa forma, é possível determinar o melhor modelo que se adequa às variáveis explicativas. Os modelos espaciais utilizados foram o Spatial Lag Model (SLM) e o Spatial Error Model (SEM) (Anselin, 2005).

A normalidade dos erros foi verificada por meio do teste de Jarque-Bera. Um pressuposto que deve ser atendido pelo modelo de regressão múltipla é a independência dos resíduos, o que raramente acontece quando se utiliza dados espaciais, pela observação de tendência nos dados ou presença de autocorrelação espacial. E neste caso, o fator espacial deve ser incorporado no modelo, uma vez que a dependência entre observações altera o poder explicativo do mesmo (Jerrett et al., 2010). Para isto, é necessário a incorporação da matriz de vizinhança, $W$, que é uma ferramenta básica para estimar variabilidade espacial de dados de área.

A determinação do melhor modelo foi realizada de acordo com os valores do Log da Verossimilhança, Critério de informação Akaike (AIC) e o Critério Bayesiano de Schwarz (Anselin, 2005).

O índice de Moran dos resíduos foi avaliado para verificar se a autocorrelação espacial foi eliminada com a aplicação dos modelos. As análises foram realizadas nos softwares TerraView 4.2.2 e GeoDa 1.12. 1.161. As figuras foram desenvolvidas no software QGIS 2.18.19 e GeoDa 1.12.1.161 (Geodacenter, 2020; Qgis, 2020).

O estudo foi submetido e aprovado pelo Comitê de Ética em Pesquisa da Universidade da Região de Joinville com protocolo n.1288516.3.0000.5366.

\section{Resultados}

Em Santa Catarina foram notificados 18.606 casos novos de tuberculose, no período entre janeiro de 2005 e dezembro de 2015, com uma média de 1.691 casos por ano. 


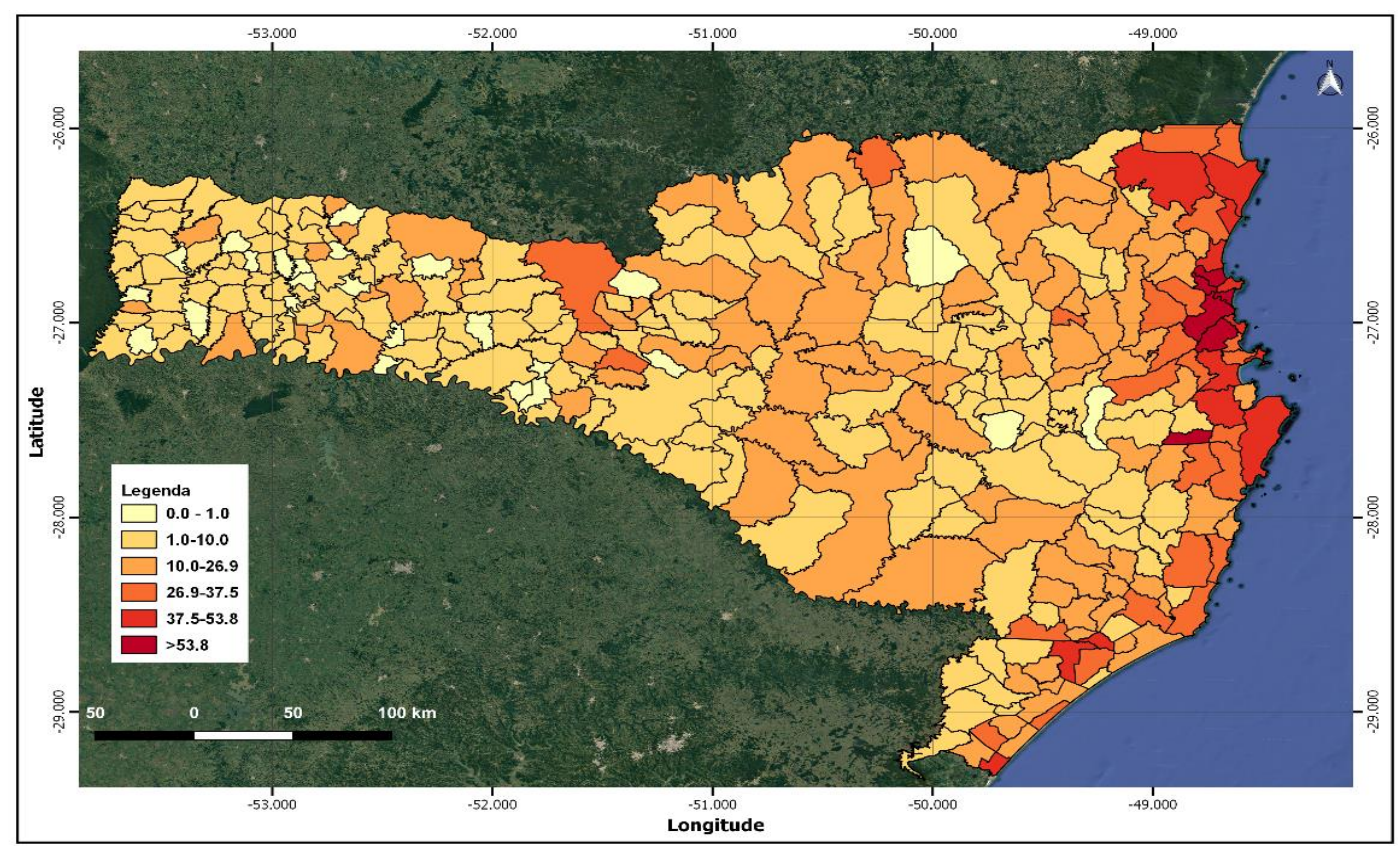

Figura 1. Mapa da distribuição das taxas brutas da média de incidência de tuberculose em Santa Catarina período 2005-2015.

Nos mapas temáticos com distribuição das taxas brutas (Figura 1) e após suavização bayesiana (Figura 2), observa-se uma concentração de incidência maior de tuberculose, numa faixa que

ocupa a face leste do estado, a faixa litorânea, que se estende da região nordeste indo até a Grande Florianópolis, tornando-se irregular em direção ao sul e numa distribuição variável, com taxas mais baixas, em direção ao interior do estado

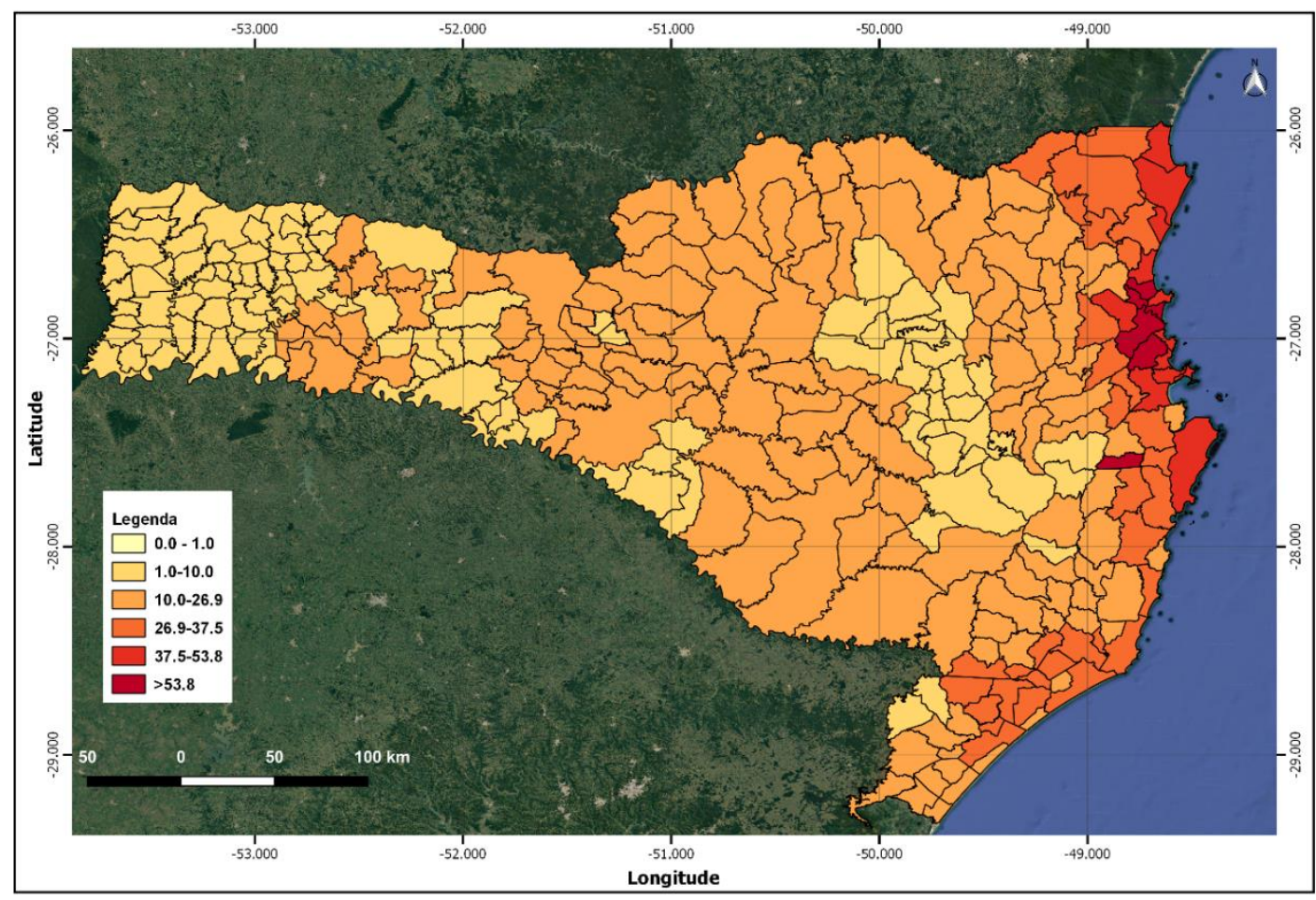

Figura 2. Mapa da distribuição das taxas bayesianas (suavizadas) medias da incidência de tuberculose período 2005-2015. 
A presença de autocorrelação espacial da variável incidência de tuberculose no estado foi verificada através do índice de Moran Global das taxas brutas $(I=0,294993, p=0,001)$.

Observa-se que a dependência espacial aumenta quando se utiliza as taxas suavizadas, com índice de Moran I de 0,336833. Na análise de autocorrelação local (LISA) taxas suavizadas (bayesianas), observou-se que localmente persistiu a presença de autocorrelação espacial com aglomerados de taxas altas e alta, "hotspots," na faixa litorânea nordeste e centro, e na região sul. Também de taxas baixas e baixas, "cold spots" para o interior do estado, e um de baixa e alta contíguo à primeira, que são vistos no mapa do espalhamento do LISA, com significância de $\mathrm{p}=0,05$ (Figura 3).

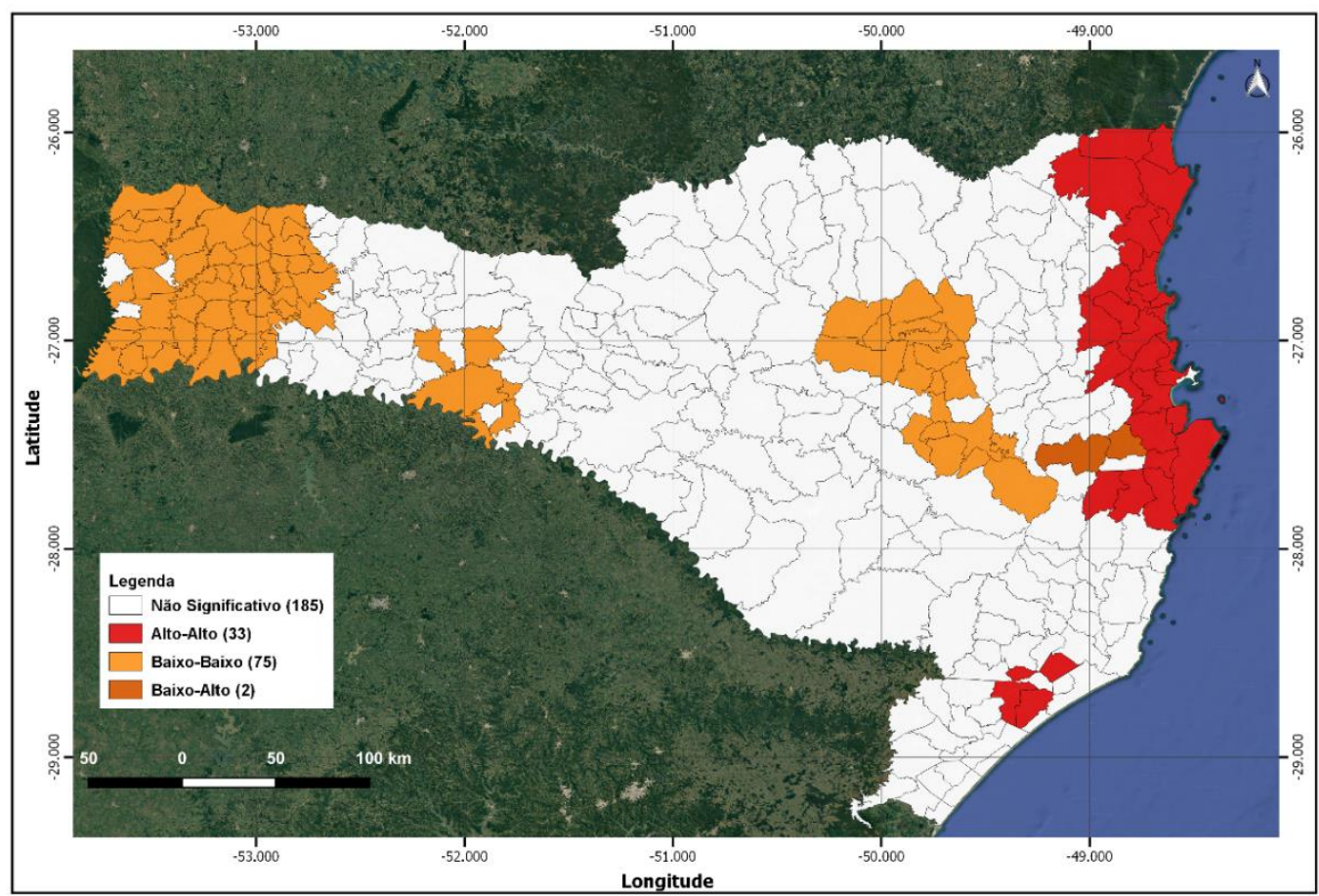

Figura 3. Mapa do índice Local de Moran (LISA) taxas bayesianas (suavizadas) da média da incidência de tuberculose em Santa Catarina período 2005-2015.

Foram identificados 6 aglomerados ou clusters com autocorrelação espacial de incidência de tuberculose significativos, 5 positivos, sendo que 2 com taxas elevadas, alto-alto, 3 com taxas baixas baixo-baixo e 1 negativo, baixo-alto. Os demais municípios não alcançaram relevância estatística de intervalo de confiança de $95 \%$, não significando, porém, que não apresentem incidência elevada de tuberculose, uma vez que a autocorrelação espacial mede a relação do atributo com sua vizinhança.

No primeiro cluster alto-alto estão agrupados 29 municípios que ocupam a faixa litorânea do estado abrangendo parte região de saúde Nordeste, passando pela região de saúde da Foz do Rio Itajaí e parte da região de saúde do Médio Vale, alcançando e abrangendo a parte leste da região da Grande Florianópolis. O segundo cluster alto-alto abrange três municípios da região carbonífera no Sul do estado.

$\mathrm{O}$ primeiro cluster baixo-baixo compreende 19 municípios da região de saúde do médio Vale do Rio Itajaí, e pequena parte contígua da região de saúde da Grande Florianópolis.

O segundo cluster baixo-baixo compreende 6 municípios da região de saúde do Alto Uruguai Catarinense. O terceiro cluster baixobaixo compreende 50 municípios, envolvendo as regiões de saúde do Extremo Oeste, região de saúde do Oeste e parte da região de Xanxerê.

$\mathrm{O}$ cluster baixo-alto compreende 2 municípios da Região da Grande Florianópolis (área de transição).

Como não foram considerados os municípios vizinhos fora da área do estado de Santa Catarina, é possível que existam regimes de autocorrelação espacial não identificados, com 
municípios do estado do Paraná, do Rio Grande do Sul e mesmo da Argentina.

As medidas descrivas das variáveis do estudo encontram-se na tabela 1.

Como resultado da correlação de Spearman/Pearson observou-se que as seguintes variáveis apresentaram correlação significativa $p<$ 0,05 e $\mathrm{R}<0,7 \mathrm{com}$ a taxa de incidência de tuberculose (com a ressalva feita para ao índice de Gini: densidade demográfica $(\mathrm{p}=0,0000)$, incidência de HIV $(p=0.0000)$, proporção de pessoas com 18 anos ou mais sem o fundamental completo e em ocupação informal $(\mathrm{p}=0,0000)$, proporção da população em domicílios com abastecimento de água e esgotamento sanitário inadequados $(\mathrm{p}=0,0012)$, taxa de mortalidade infantil $\quad(p=0,0000)$, razão de dependência $(0,0006)$, taxa de envelhecimento $(\mathrm{p}=0,0000)$, proporção de pessoas de 15 a 24 anos que não estudam nem trabalham e são vulneráveis à pobreza, proporção de mães chefes de família sem ensino fundamental completo e com filhos menores de 15 anos, proporção de vulneráveis à pobreza $(\mathrm{p}=0,0000)$, proporção da população residente na área urbana $(\mathrm{p}=0,0000)$, média do percentual de cobertura da Estratégia de Saúde de Família no período $(\mathrm{p}=0.0000)$, proporção de aumento da população $(\mathrm{p}=0,0000)$, proporção da população vivendo em domicílios com densidade superior a 2 pessoas por dormitório $(\mathrm{p}=0,0000)$, taxa de desocupação 18 anos ou mais $(\mathrm{p}=0,0000)$ e índice Gini $(\mathrm{p}=0,0842)$.

O modelo de regressão teve como ajuste melhor final as variáveis: índice de Gini (correlação negativa), proporção da população vivendo em domicílios com densidade superior a 2 pessoas por dormitório, proporção de pessoas de 15 a 24 anos que não estudam nem trabalham e são vulneráveis à pobreza, taxa de desocupação 18 anos ou mais (desemprego), incidência média de HIV no período e aumento proporcional da população (Tabela 2).

O índice de Moran para os resíduos da análise de regressão clássica foi de 0,414 ( $\mathrm{p}<$ 0,001 ), o que demonstrou a presença de auto correlação espacial dos resíduos.

A comparação dos modelos da regressão clássica e com fator espacial SEM e SLM encontram-se na tabela 3.

\section{Discussão}

Este estudo mostra que a distribuição dos casos de tuberculose em Santa Catarina não é homogênea e nem obedece a um padrão aleatório. A concentração na região Nordeste e na faixa litorânea tem o padrão da ocupação histórica do estado, com incidência maior nas regiões ou municípios mais antigos e mais populosos, que é um reflexo da ocupação e do perfil socioambiental históricamente construídos no território brasileiro (de Souza et al., 2020). Este gradiente leste oeste da distribuição da tuberculose está de acordo com um estudo sobre análise espacial de determinantes socioeconômicos da tuberculose no Brasil, onde encontraram forte correlação espacial, notavelmente em áreas urbanas, da costa leste do país e na fronteira oeste (Garcia e Silva, 2016) e tsmbém das desigualdades regionais na oferta de saúde (De Albuquerque et al., 2017).

Quando se analisa as taxas de incidência após o alisamento bayesiano, o padrão de maior incidência da face leste do estado permanece evidente. Existem evidências de que as taxas de notificação de TB aumentam com a temperatura e diminuem com a altitude, o que poderia explicar essa concentração maior no litoral (Gelaw et al., 2019).

A suavização bayesiana procura corrigir distorções das pequenas taxas, também tenta corrigir eventuais subnotificações, subdiagnósticos ou registros em município que não seja o de origem do paciente. Isso se deve ao fato de que o fluxo de pessoas e fluxo de atendimento em saúde normalmente tende dos municípios menores para o município sede da região, por razões diversas, mas principalmente pela concentração de recursos. Em Santa Catarina, no período 2005-2015, 80\% dos casos de tuberculose se concentraram em 33 municípios, que correspondem a $11,2 \%$ do total dos municípios, a maioria deles na faixa litorânea, onde se concentram os municípios com densidade demográfica mais elevada. Com relação à incidência, 166 municípios não notificaram ou apresentaram incidência baixa de tuberculose e 252 municípios notificaram abaixo da média do estado. Incidências acima da média brasileira ocorreram em 18 municípios, sendo as maiores incidências em Balneário Piçarras, Camboriú, Itajaí, Navegantes e São Pedro de Alcântara.

Pelo cálculo do índice de Moran global foi identificada autocorrelação espacial positiva, indicando que a tuberculose tem uma distribuição no estado que apresenta dependência espacial, ou seja, que no estado a ocorrência da tuberculose é dependente de sua ocorrência nas áreas vizinhas. A dependência espacial tende a aumentar um pouco quando se utiliza as taxas suavizadas, apesar disso, as vantagens da utilização deste método superam este viés e são recomendadas (Magalhães e Medronho, 2017). 
Tabela 1 - Medidas descritivas

\begin{tabular}{|c|c|c|c|}
\hline Variáveis & Média \pm DP & $($ Mediana \pm DQ $)$ & Amplitude \\
\hline \multicolumn{4}{|l|}{ Indicadores demográficos } \\
\hline $\begin{array}{l}\text { Estimativa do aumento } \\
\text { proporcional da população em } \\
\text { relação ao censo de } 2000\end{array}$ & $(16,85 \pm 12,81)$ & $(13,93 \pm 8,55)$ & $(-38,09-57,27)$ \\
\hline Densidade Demográfica & $(82,22 \pm 190,41)$ & $(31,97 \pm 21,48)$ & $(2,06-2337,67)$ \\
\hline $\begin{array}{l}\text { Proporção da população } \\
\text { residente na área urbana }\end{array}$ & $(59,07 \pm 23,9)$ & $(57,5 \pm 21,35)$ & $(14,1-100)$ \\
\hline Taxa de envelhecimento & $(8,24 \pm 1,81)$ & $(8,27 \pm 1,21)$ & $(4,13-13,57)$ \\
\hline Razão de dependência & $(44,42 \pm 4,88)$ & $(44,15 \pm 3,21)$ & $(29,34-63,57)$ \\
\hline \multicolumn{4}{|l|}{ Indicadores Socioeconômicos } \\
\hline Índice GINI & $(0,44 \pm 0,05)$ & $(0,44 \pm 0,04)$ & $(0,28-0,62)$ \\
\hline $\begin{array}{l}\text { Proporção de vulneráveis à } \\
\text { pobreza }\end{array}$ & $(18,29 \pm 11,02)$ & $(15,3 \pm 7,69)$ & $(3,45-48,16)$ \\
\hline $\begin{array}{l}\text { Proporção da população em } \\
\text { domicílios com abastecimento } \\
\text { de água e esgotamento sanitário } \\
\text { inadequados }\end{array}$ & $(1,69 \pm 2,84)$ & $(0,78 \pm 0,87)$ & $(0,01-28,44)$ \\
\hline 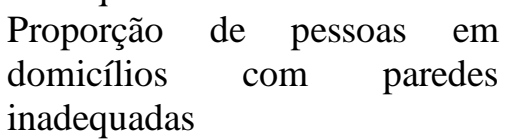 & $(2,02 \pm 2,35)$ & $(1,27 \pm 0,92)$ & $(0,05-19,27)$ \\
\hline $\begin{array}{l}\text { Proporção da população } \\
\text { vivendo em domicílios com } \\
\text { densidade superior a } 2 \text { pessoas } \\
\text { por dormitório }\end{array}$ & $(12,15 \pm 5,25)$ & $(11,05 \pm 3,05)$ & $(4,16-43,65)$ \\
\hline $\begin{array}{l}\text { Proporção de pessoas de } 15 \text { a } 24 \\
\text { anos que não estudam nem } \\
\text { trabalham e são vulneráveis à } \\
\text { pobreza }\end{array}$ & $(28,35 \pm 11,57)$ & $(28,99 \pm 7,24)$ & $(0-60,25)$ \\
\hline $\begin{array}{l}\text { Proporção de mães chefes de } \\
\text { família sem ensino fundamental } \\
\text { completo e com filhos menores } \\
\text { de } 15 \text { anos }\end{array}$ & $(48,26 \pm 12,13)$ & $(47,73 \pm 8,43)$ & $(23,19-88,38)$ \\
\hline $\begin{array}{l}\text { Proporção de pessoas com } 18 \\
\text { anos ou mais sem o fundamental } \\
\text { completo e em ocupação } \\
\text { informal }\end{array}$ & $(38,18 \pm 10,18)$ & $(36,92 \pm 7,11)$ & $(12,5-66,25)$ \\
\hline $\begin{array}{l}\text { Índice de Desenvolvimento } \\
\text { Humano municipal }\end{array}$ & $(0,73 \pm 0,04)$ & $(0,74 \pm 0,03)$ & $(0,62-0,85)$ \\
\hline $\begin{array}{l}\text { Produto Interno Bruto por } \\
\text { município }\end{array}$ & $(524662,15 \pm 1613575,06)$ & $(121323 \pm 144635,5)$ & $(17048-18252540)$ \\
\hline $\begin{array}{l}\text { Taxa de desocupação } 18 \text { anos ou } \\
\text { mais }\end{array}$ & $(2,48 \pm 1,79)$ & $(2,02 \pm 1,16)$ & $(0,11-14,7)$ \\
\hline
\end{tabular}

\section{Indicadores de saúde}

Incidência de Tuberculose no período

$(59,07 \pm 23,9)$

$$
(57,5 \pm 21,35)
$$$$
(14,1-100)
$$$$
(8,24 \pm 1,81)
$$$$
(8,27 \pm 1,21)
$$$$
(4,13-13,57)
$$$$
(29,34-63,57)
$$

\section{Índice GIN}

$(18,29 \pm 11,02)$

$(3,45-48,16)$

$(1,69 \pm 2,84)$

$(0,78 \pm 0,87)$

$(0,01-28,44)$

inadequados

Proporção de pessoas em inadequadas

$(2,02 \pm 2,35)$

$(1,27 \pm 0,92)$

$(0,05-19,27)$

$(12,15 \pm 5,25)$

$(11,05 \pm 3,05)$

$(4,16-43,65)$

$(28,35 \pm 11,57)$

$(28,99 \pm 7,24)$

$(0-60,25)$

$(48,26 \pm 12,13)$

$(47,73 \pm 8,43)$

$(23,19-88,38)$

Taxa de mortalidade Infantil

$\begin{array}{ccc}(14,03 \pm 19,48) & (9,28 \pm 6,63) & (0-272,5) \\ (12,59 \pm 2,42) & (12,2 \pm 1,85) & (8,55-19,9) \\ (15,86 \pm 15,74) & (12,27 \pm 7,35) & (0-63,78)\end{array}$

HIV Incidência de HIV no período

$(15,86 \pm 15,74)$

$(12,27 \pm 7,35)$

$(89,23 \pm 16,73)$

$(97,18 \pm 7,13)$

$(24,21-100)$

Média do percentual de cobertura da Estratégia de Saúde de Família no período

I - DP: desvio padrão; DQ: desvio quartílico. 
Tabela 2. Modelo OLS para o log da taxa de incidência após alisamento bayesiano

\begin{tabular}{lrrrr}
\hline Variável & Coeficiente & Erro Padrão & $\mathrm{t}$ & $\mathrm{p}$ \\
\hline Índice de Gini & $-1,96906$ & 0,513376 & $-3,83551$ & $<0,001$ \\
\% de domicílio com mais de 2 & 0,01366 & 0,005359 & 2,54908 & 0,011 \\
pessoas/ dormitório & & & & \\
Taxa de desocupação em & 0,09579 & 0,017913 & 5,34762 & $<0,001$ \\
população de 18+ anos & & & & \\
Vulneráveis 15 a 24 anos & 0,00642 & 0,002514 & 2,55418 & 0,011 \\
Incidência HIV & 0,02534 & 0,002932 & 8,64180 & $<0,001$ \\
Aumento População & 0,00524 & 0,001727 & 3,03226 & 0,003 \\
\hline
\end{tabular}

Tabela 3. Coeficiente de determinação, log da verossimilhança, critério de informação de Akaike e Critério Bayesiano de Schwarz Dos modelos OLS, SLM, SEM.

\begin{tabular}{lrrr}
\hline Variável & OLS & SLM & SEM \\
\hline $\mathrm{R}^{2}$ & 0,606 & 0,808 & 0,804 \\
Log da Verossimilhança & $-164,220$ & $-75,510$ & $-89,594$ \\
$\mathrm{AIC}$ & 342,245 & 167,019 & 193,188 \\
$\mathrm{SBC}$ & 367,837 & 196,267 & 218,780 \\
Moran - (Resíduo) & $0,414 *$ & $0,108^{*}$ & $0,113 *$ \\
\hline${ }^{*} \mathrm{p}<0,05$ & & &
\end{tabular}

Pelo cálculo do índice de Moran Local (LISA) e sua representação cartográfica, foi possível observar o comportamento desigual da doença, com áreas de aglomerados de alta e baixa incidência, estatisticamente significativos.

No presente estudo não foram considerados os municípios vizinhos fora da área do Estado de Santa Catarina, sendo possível existirem regimes de autocorrelação espacial não identificados com municípios do Estado do Paraná, do Rio Grande do Sul e, mesmo, da Argentina.

A questão da tuberculose em áreas de fronteiras é um problema reconhecidamente relevante pelo trânsito de pessoas e registros em sistemas diferentes e atendimento de saúde distintos bem como de perfil de resistência aos medicamentos (Desisto et al., 2015). Apesar da tuberculose ser doença curável, e contar com estratégias eficazes para seu controle e eliminação, ainda apresenta elevada taxa de mortalidade, presença de doenças associadas e vulnerabilidade em áreas de fronteira (Dara et al. 2017; Silva et al., 2019).

Vários estudos identificam a tuberculose como uma doença com autocorrelação espacial positiva (Wubuli et al., 2015; Rao et al. 2016; Zhang, 2017). O que possibilitou identificar áreas de maior carga de tuberculose e áreas de maior chance de transmissão e, assim, obter melhor compreensão da dinâmica da doença, também compreender que a incidência em determinada cidade era influenciada pela incidência das cidades vizinhas, implicando que a transmissão recente possa ter importância na endemia de tuberculose. Estudo na província de Shandong, China, identificou áreas de hotspots, de tuberculose e áreas de maior chance de 
transmissão (Zhang, 2017). Tambem estudos de análise espacial permitiram encontrar associação de aumento da incidência de tuberclose em áreas de maior poluição ambiental e atmosférica (Carrasco-Escobar et al., 2020, Liu et al., 2020).

Apesar da tuberculose ser uma doença de forte associação com fatores socioeconômicos ligados à pobreza, no estado de Santa Catarina observa-se um padrão de distribuição espacial que se superpõe ao das áreas de maior desenvolvimento econômico dentro do estado (Secretaria do Estado da Fazenda, 2016). Essa situação contrasta com os achados para o Brasil, em dados recentes do Ministério da Saúde, onde o coeficiente de incidência de tuberculose nos municípios com melhores condições socioeconômicas aumentou em $1,8 \%$, passando de 31,8/100 mil habitantes em 2015, para 32,3/100 mil hab. em 2018, já um aumento de $2,7 \%$ foi observado nos municípios com piores condições socioeconômicas, tendo o coeficiente evoluído de 52,2/100 mil hab. em 2015 para 53,7/100 mil hab. em 2018 (Ministério da Saúde, 2019 ( Brasil, 2019).

Segundo análise da Diretoria de Planejamento Orçamentário de Santa Catarina, onde são comparados 10 fatores de competitividade (Secretaria do Estado da Fazenda, 2016), observou-se que nas regiões com melhores índices estão concentradas as maiores taxas de incidência de tuberculose do estado, ou seja, onde estão as melhores condições de vida e de oferta de serviços de saúde.

Junto ao primeiro cluster alto-alto, o maior, é evidenciada uma pequena área correspondente ao município de São Pedro de Alcântara com a incidência mais elevada do estado, que se destaca nos mapas de taxas brutas e bayesianas, porém, com pequena população, onde se localiza o Complexo Prisional Estadual. Embora nitidamente ocupe uma posição de outlier na distribuição e não tenha alcançado significância de autocorrelação espacial, foi mantido na discussão para ressaltar o problema da tuberculose prisional cujas taxas de incidência, mesmo no seu entorno, são muito superiores à população geral (Sánchez e Larouzé, 2016; Valença et al., 2016), especialmente correlacionado com a superpopulação carcerária (Simpson et al., 2019).

$\mathrm{Na}$ análise de regressão múltipla e considerando o fator espacial, no modelo com melhor ajuste, encontramos o índice de Gini com correlação negativa. Este resultado é aparentemente incongruente, mas no estado a maior ocorrência de tuberculose ocorre justamente onde o índice é menor, ou seja menor desigualdade. Possivelmente isso se deva pelo densidade demográfica, presença de aglomerados urbanos subnormais e fluxo migratório maiores nestas regiões. Essa situação foi identificado em estudo realizado em Curitiba (PR), que apresenta padrão socioeconômico elevado e apresenta áreas de risco elevado para adoecimento e morte por tuberculose, revelando a iniquidade social em áreas mais carentes dentro do município (Dos Santos et al., 2020).

A ocorrência da tuberculose, em áreas não necessariamente com condições de vida mais precárias não é incomum. Estudo em São Carlos (SP), demonstrou que as regiões de maior risco de adoecimento, eram as de maior densidade demográfica e de maior variação de características popullacionais e de ocupação urbana, sendo as regiõs de aglomerados de mais alto risco apresentavam vulnerabilidade social média (Arroyo et al., 2017).

Em análise espacial da tuberculose pulmonar no Rio de Janeiro, encontrou-se clusters significativos de alta incidência no litoral sul e de baixa incidência no norte do estado, concluindo que as megacidades são importantes áreas de risco para ocorrência da tuberculose (Rodrigues, et al., 2017). Presença de grandes clusteres contribuem significativamente para o aumento da carga de tuberculose (De Abreu E Silva et al., 2016).

Outro indicador correlacionado à incidência da doença no presente estudo foi o aumento populacional. Sabe-se que grandes populações e áreas de grande circulação de pessoas estão relacionadas à incidência aumentada de tuberculose (Izumi et al., 2015). A distribuição espacial da doença tende a ser desigual nas diversas regiões e diversos municípios, a incidência é maior nas regiões com maior densidade demográfica, precárias condições de vida e onde o fluxo de pessoas é intenso. E modificação dessa dinâmica pode concorrer para a mitigação do problema (Ortblad et al., 2015).

Enquanto o Brasil teve um aumento populacional entre 1990 e 2016 de 40\%, Santa Catarina aumentou 52\% (Ministério do Planejamento Orçamento e Gestão, 2018; Programa das Nações Unidas para o Desenvolvimento, Instituto de Pesquisa Econômica Aplicada, 2012). Quando se analisa o aumento populacional entre o censo de 2000 e a projeção para 2016, o Brasil teve um aumento de 
17,6\% e Santa Catarina 19,9\%. Ao comparar os estados com maior intercâmbio populacional com Santa Catarina destacam-se Rio Grande do Sul e Paraná, respectivamente com um incremento populacional de $9,7 \%$ e $14,9 \%$.

No comparativo do aumento populacional das capitais, Florianópolis aumentou $28 \%$, Curitiba $16,2 \%$ e Porto Alegre $8,1 \%$. O Paraná apresenta uma situação epidemiológica da tuberculose melhor que a de Santa Catarina, porém, no Rio Grande do Sul a situação é pior. Neste último, a maior parte da perda populacional ocorreu com trocas migratórias com os estados da Região Sul, sendo $87 \%$ com o estado de Santa Catarina (Bandeira et al., 2014). Em algumas cidades de Santa Catarina em que a taxa de incidência de tuberculose é mais elevada, o incremento populacional foi maior ainda, como São José 26,5\%, Itajaí 29,4\%, Joinville 24,6\%, Balneário de Camboriú 44,2\%, para citar algumas (Ministério do Planejamento Orçamento e Gestão, 2018).

Como Santa Catarina tem uma das menores taxas de fecundidade do país, apesar da menor taxa de mortalidade infantil, o aumento populacional deve ter ocorrido em consequência de ondas de migração. Entre 1995 e 2010, o estado teve um saldo positivo de migrantes (Ministério do Planejamento, 2012). De fato, a região Sul, graças ao desempenho de Santa Catarina, recebeu um volume 59\% maior de imigrantes, tendo sido a única região com aumento da mobilidade espacial da população no quinquênio 2005-2010 (Ministério do Planejamento, 2012). E com relação à tuberculose, os movimentos migratórios são um importante fator epidemiológico na cadeia de transmissão (Pareek et al. 2016, Liao et al., 2020). Em geral os movimentos migratórios buscam as áreas metropolitanas onde alcançam melhores condições de vida (Badmus et al., 2016).

$\mathrm{O}$ conceito de aglomerados subnormais abarca uma diversidade de assentamentos irregulares, incluindo favelas, invasões, grotões, baixadas, comunidades, vilas, ressacas, mocambos, palafitas e outros (Ministério do Planejamento Orçamento e Gestão, 2011). O aumento de aglomerados espaciais, bem como de sua população, contribui para a disseminação e perenização da doença numa determinada região (Bergonzoli et al., 2016; Desaleng e Koya, 2015). Além disso outras consequências de um rápido crescimento demográfico e espacial são a alta criminalidade, tráfico de drogas, favelização, pobreza, degradação ambiental, desemprego, contribuindo para uma piora da condição de vida e de saúde. A coexistência de desnutrição, dificuldade de acesso à serviços de saúde, convivência em ambientes mal ventilados e com superlotação de pessoas, aumenta muito o risco de contágio e desenvolvimento da tuberculose (Badmus et al., 2016). A pobreza em si, é um dos fatores mais importantes na cadeia de transmissão da tuberculose(Sales et al., 2015).

Estudo no município de Itaboraí (RJ) encontrou que o percentual de abandono e óbito, bem como de menor percentual de cura é duas vezes maior nas áreas de maior vulnerabilidade social (Alves Filho et al., 2017).

Um estudo realizado na Índia, onde a tuberculose é um grave problema de saúde pública nas áreas urbanas, demonstrou que apesar das áreas urbanas proporcionarem uma melhor oferta em termos de saúde pública, o impacto quanto à tuberculose nem sempre é positivo. A incidência relativa de tuberculose em áreas urbanas é 1,69 vezes maior do que nas áreas rurais na Índia (Prasad et al., 2016). E estudo realizado também nesse país, concluiu que desnutrição, viver em favelas, poluição do ar interior e ingestão de álcool foram encontrados como fatores de risco modificáveis para tuberculose (Dhanaraj et al., 2015).

Em um estudo acerca da ocorrência de tuberculose na União Europeia, verificou-se que, em 15 grandes cidades de países com baixa incidência, as taxas de notificação eram o dobro da taxa da nação correspondente (Vries et al., 2015).

A questão da tuberculose é indissociável da discussão sobre iniquidade social e vulnerabilidade, comuns nas grandes cidades do Brasil. A tuberculose no Brasil acomete especialmente a faixa etária de população jovem, na fase de vida mais produtiva, sendo $86,6 \%$ dos casos entre 15 e 64 anos e $41,5 \%$ entre 15 e 34 anos (Ministério da Saúde, 2017). Análise espaço temporal realizada em área de iniquidade social, no nordeste do Brasil, demonstrou um aumento no acometimento de pessoas mais jovens, predominando sexo masculino abaixo de 20 anos e entre 20 a 39 anos de idade, com baixa taxa de cura (Verônica et al., 2019).

Em análise da distribuição espacial da tuberculose em Manaus, demonstrou-se que a média de pessoas por dormitório, desemprego e proporção de casas não ligada à sistema de esgoto foram preditores de tuberculose (Castro et al., 2018). Em trabalho realizado em Portugal, correlacionando tuberculose com iniquidade 
social encontrou que desemprego e trabalho braçal eram preditores para doença (Apolinário et al., 2017).

Sabe-se que a transmissão da tuberculose é maior em locais de aglomeramento de pessoas, populações de instituições fechadas como asilos e presídios. Por ser uma doença infecciosa, de contaminação e disseminação aérea, ocorre mais em ambientes onde o doente vive e coabita com pessoas suscetíveis de serem contaminadas. O risco de contato íntimo com paciente tuberculoso, de adquirir a doença é elvado (Reichler et al., 2018).

O fator mais significativo no aumento do número de casos de tuberculose, num estudo da distribuição espacial, realizado em cidade de porte médio no Brasil, foi a densidade populacional da área do município (De Abreu $\mathrm{E}$ Silva et al., 2016). Lembrrando que as cidades de Santa Catarina em geral são de porte médio.

No presente estudo, no modelo final de regressão a incidência de tuberculose ficou correlacionada à variável proporção de domicílios com mais de duas pessoas por dormitório, vulnerabilidade social e desemprego.

Outro indicador importante na cadeia de transmissão e manutenção da doença é sua relação com a infecção pelo HIV, que também foi identificado, no modelo final do de regressão do presente trabalho, como fator significativo. A coinfecção TB/HIV é um importante fator na manutenção, disseminação e fator de insucesso para o tratamento da tuberculose (Duarte et al., 2018; Teixeira De Siqueira-Filha et al., 2018).

É fator de vulnerabilidade na definição dos subcenários de gravidade no programa nacional para o fim da tuberculose (Ministério da Saúde, 2017). O Brasil, na presente distribuição entre os 30 países com maior carga de tuberculose, faz parte do subcenário com incidência elevada de HIV. A região sul, particularmente Santa Catarina e Rio Grande do Sul, apresentam as maiores incidências de HIV. Em estudo da distribuição espacial da AIDS e determinantes sociais de saúde realizado no Ceará, encontrou que as taxas mais elevadas de AIDS foram encontradas nos municípios que apresentaram melhores condições de vida (M. da S. Lima et al., 2016).

$\mathrm{O}$ modelo de regressão encontrou coeficiente de determinação com índice de Gini com uma correlação negativa, e correlações positivas com taxa de desocupação de pessoas com 18 anos ou mais, percentual de pessoas de 15 a 24 anos que não estudam, não trabalham e são vulneráveis na população nesta faixa etária, percentual de domicílios com mais de duas pessoas por dormitório, aumento da população e incidência de HIV. Teve como output um coeficiente de determinação de 0,606 , e quando é agregado o fator espacial nos modelos SEM $\left(\mathrm{R}^{2}\right.$ $=0,804)$ e SLM $\left(R^{2}=0,808\right)$ obtém-se um coeficiente de determinação maior, inferindo que com a agregação do fator espacial a acurácia do modelo aumenta. O índice de Moran dos resíduos, apesar de reduzir bastante não foi eliminado, o que pode sugerir que estes indicadores possuam autocorrelação espacial independentemente de sua associação com a incidência de tuberculose.

Estudo realizado em 2017 sobre a incidência de tuberculose no Brasil correlacionando com diversos indicadores socioeconômicos e de saúde, encontrou variação regional e também associação com fatores como AIDS, proporção de população privada de liberdade, renda familiar per capita, aglomeração dentro dos domicílios, proporção de pobres e vulneráveis, taxa de desemprego e cobertura da atenção básica (Pelissari e Diaz-Quijano, 2017).

Estes achados são semelhantes aos encontrados no presente estudo, com a diferença da associação inversa com o índice de Gini, que pode ser por conta da variação regional, de não termos conseguido demonstrar correlação com a cobertura pela Estratégia de Saúde de Família, (ESF) e de não termos estudado população prisional.

\section{Conclusão}

A distribuição espacial da tuberculose no estado de Santa Catarina entre os anos de 3005 a 2015 mostra a existência de autocorrelação espacial. A doença tnde a se concentrar nos conglomerados urbanos das maiores cidades, que no estado, são áreas com aumento populacional recente, receptoras de fluxo migratório e com formação de aglomerados urbanos subnormais.

A correlação com indicadores socioeconômicos, demográficos e de saúde mostra a presença de pessoas suscetíveis a desenvolverem a doença, especialmente jovens na faixa etária produtiva e em condição de vulnerabilidade social. Houve correlação inversa entre a incidência de tuberculose e o índice de Gini, e correlação direta com a taxa de desocupação de 18 anos ou mais, percentual de pessoas de 15 a 24 anos vulneráveis, domicílios 
com mais de duas pessoas por dormitório, aumento da população e incidência de HIV.

Seria oportuno estender o estudo, especialmente nos municípios dos clusters com autocorrelação espacial alta, num nível mais detalhado de análise, com polígonos ou áreas menores, como bairros ou setores censitários, para identificar onde se encontram as áreas de maior concentração ou bolsões da doença nestes municípios. Esta informação possibilitaria políticas de saúde mais intensivas e focadas na tuberculose e seus determinantes nestes locais.

\section{Agradecimentos}

À Equipe do Programa de Controle da Tuberculose da Diretoria de Vigilância Epidemiológica da Secretaria de Saúde do Estado de Santa Catarina pela orientação na coleta e interpretação dos dados e à Coordenação do programa de Pós-graduação em Saúde e Meio Ambiente da Univille.

\section{Referências}

Abreu D.M.X. de, Pinheiro P.C., Queiroz B.L., Lopes, É.A.S., Machado A.T.G.M; Lima, A.M. de LD de, et al., 2018. Análise espacial da qualidade da Atenção Básica em Saúde no Brasil. Saúde em Debate. 42(spe1):67-80. Disponível:

https://www.scielo.br/scielo.php?script=sci_a rttext\&pid=S0103-11042018000500067. Doi 10.1590/0103-11042018S105. Acesso: 28 jun. 2020.

Alves Filho, P., Pellegrini Filho, A., Ribeiro, P. T., de Toledo, L. M., Romão, A. R., \& Novaes, L. C. M., 2017. Desigualdades socioespaciais relacionadas à tuberculose no município de Itaboraí, Rio de Janeiro. Revista Brasileira de Epidemiologia, 20(4), 559-572. Doi: 10.1590/1980-5497201700040001.

Anselin L., 2005. Exploring spatial data with GeoDa TM: a workbook. Center for Spatially Integrated Social Science [online]. Disponível:

http//:csiss.org/clearinghouse/GeoDa/geodaw orkbook.pdf. Acesso: 22 fev. 2017.

Anselin, L., 1995. Local indicators of spatial association-LISA. Geographical Analysis, 27(2), 93-115. Availabe: https://onlinelibrary.wiley.com/doi/epdf/10.1 111/j.1538-4632.1995.tb00338.x. Access: Feb $22 \quad 2017$. Doi: $\quad 10.1111 / \mathrm{j} .1538$ 4632.1995.tb00338.x.

Apolinário, D., Ribeiro A.I., Krainski E., Sousa P., Abranches M., Duarte R.., 2017. Tuberculosis inequalities and socio-economic deprivation in Portugal. Int J Tuberc Lung Dis. 21(7):6. Available: http://dx.doi.org/10.5588/ijtld.16.0907. Access: Jun 282020.

Arroyo, L. H., Yamamura, M., Protti-Zanatta, S. T., Fusco, A. P. B., Palha, P. F., Ramos, A. C. V., Uchoa, S. A., \& Arcêncio, R. A., 2017. Identificação de áreas de risco para a transmissão da tuberculose no município de São Carlos, São Paulo, 2008 a 2013. Epidemiologia e Servicos de Saude: Revista Do Sistema Unico de Saude Do Brasil, 26(3), 525-534. Doi: 10.5123/S167949742017000300010.

Badmus, O., Camorlinda, S., \& Simpson, O., 2016. Poverty and the emergence of tuberculosis: An agent-based modelling approach. Disponível: https://www.researchgate.net/publication/301 913250_Poverty_and_the_emergence_of_tub erculosis_An_agent-

based_modelling_approach. Doi: 561-564. 10.1109/BHI.2016.7455959. Acess: Dec 12 2019.

Bandeira, M.D., Zuanazzi, P.T., Agranonik, M., Rauber, V., 2014. Uma análise de fluxo migratório no Rio Grande do Sul e suas mesorregiões. Indicadores Econômicos FEE. [online]. 41(4). Disponível: http://www.ilsl.br/revista/download.php?id=i mageBank/v39n1a06.pdf. Acesso: 28 set. 2017.

Barcellos, C., Ramalho, W. M., Gracie, R., Magalhães, M. D. A. F. M., Fontes, M. P., \& Skaba, D. A., 2008. Georreferenciamento de dados de saúde na escala submunicipal: algumas experiências no Brasil. Epidemiologia e Serviços de Saúde, 17(1), 59$70 . \quad$ Doi: $\quad 10.5123 /$ S167949742008000100006.

Bergonzoli, G., Castellanos, L,G,, Rodríguez, R., Garcia, L.M., 2016. Determinants of 
tuberculosis in countries of Latin America and the Caribbean. Revista Panamericana de Salud Publica. 39, 101-105.

Bonifácio, S. R., \& Lopes, E. L., 2019. Mapeamento de Agravos de Saúde: uma aplicação da técnica de georeferenciamento Com o Uso do Software Google Earth. International Journal of Health Management, 2, 1-16. Disponível: http://ijhmreview.org. Acesso: 17 jul. 2020.

BRASIL. Ministério da Saúde. Secretaria de Vigilância em Saúde. Boletim Epidemiológico., $2019 . \quad$ Disponível: https://www.saude.gov.br/images/pdf/2019/m arco/22/2019-009.pdf. Acesso: 10 jun. 2020.

BRASIL. Ministério da Saúde. Secretaria de Vigilância em Saúde. Fundação Oswaldo Cruz., 2007. Introdução à estatística espacial para a saúde pública. Brasília. Disponível: http://www.escoladesaude.pr.gov.br/arquivos /File/TEXTOS_CURSO_VIGILANCIA/capa citacao_e_atualizacao_em_geoprocessamento _em_saude_3.pdff. Acesso: 20 ago. 2019.

Carrasco-Escobar, G., Schwalb, A., TelloLizarraga, K., Vega-Guerovich, P., \& UgarteGil, C., 2020. Spatio-temporal co-occurrence of hotspots of tuberculosis, poverty and air pollution in Lima, Peru. Infectious Diseases of Poverty, 9, 1-6. doi: 10.1186/s40249-02000647-w.

De Castro, D. B., Sadahiro, M., Pinto, R. C., de Albuquerque, B. C., \& Braga, J. U., 2018. Spatial distribution of tuberculosis in Manaus and its social determinants, 2008-2013. The International Journal of Tuberculosis and Lung Disease. 22(2), 171-178. Availabe: http://dx.doi:10.5588/ijtld.17.0369. Access: Jun 202020.

Clancy, L., Rieder, H.L., Enarson, D. A., \& Spinaci S., 1991. Tuberculosis elimination in the countries of Europe and other industrialized countries. European respiriratory journal. 4(10),1288-1295.

Dara M, Sulis G, Centis R, D'Ambrosio L, De Vries G, Douglas P, et al., 2017. Cross-border collaboration for improved tuberculosis prevention \& care: policies, tools and experiences. International Journal of
Tuberculosis and Lung Disease. 21(7), 727 736.

De Abreu E Silva, M., Di Lorenzo Oliveira, C., Teixeira Neto, R. G., \& Camargos, P. A., 2016. Spatial distribution of tuberculosis from 2002 to 2012 in a midsize city in Brazil. BMC Public Health, 16(1), 1-8. doi: 10.1186/s12889-016-3575-y.

De Vries, G., Aldridge, R. W., Cayla, J. A., Haas, W. H., Sandgren, A, van Hest, N. A, et al., 2015. Epidemiology of tuberculosis in big cities of the European Union and European Economic Area Countries. Eurosurveillance, 19(6). Doi:10.2807/15607917.ES2014.19.9.20726. Access: Jun 20 2020.

Albuquerque, M. V. D., Viana, A. L. D. Á., Lima, L. D.D., Ferreira, M. P., Fusaro, E. R., \& Iozzi, F. L., 2017. Regional health inequalities: changes observed in Brazil from 2000-2016. Ciência e Saúde Coletiva, 22, 1055-1064. doi: 10.1590/1413-81232017224.26862016.

De Lima, L. M. M., de Sá, L. R., dos Santos Macambira, A. F. U., de Almeida Nogueira, J., de Toledo Vianna, R. P., \& de Moraes, R. M., 2019. A new combination rule for Spatial Decision Support Systems for epidemiology. International journal of health geographics, 18(1), 1-10. Doi: 10.1186/s12942-019-01877.

De Souza, H. P., de Oliveira, W. T. G. H., dos Santos, J. P. C., Toledo, J. P., Ferreira, I. P. S., de Sousa Esashika, S. N. G., de Lima, T. F. P., \& de Sousa Delácio, A., 2020. Doenças infecciosas e parasitárias no Brasil de 2010 a 2017: aspectos para vigilância em saúde. Revista Panamericana de Salud Pública, 44, 1. Doi: $10.26633 /$ rpsp.2020.10.

Desaleng, D., Koya, P.R., 2015. The role of polluted air and population density in the spread of Mycobacterium tuberculosis disease. J Multidiscip Eng Sci Technol. [online]. 2, 5, 1212-1220. Availabe: http://www.jmest.org/wpcontent/uploads/JMESTN42350782.pdf. Access: Sep 282017.

Desisto, C., Broussard, K., Escobedo, M., Borntrager, D., Alvarado-ramy, F., \& 
Waterman, S., 2015. Border lookout: enhancing tuberculosis control on the United States - Mexico Border. The American journal of tropical medicine and hygiene, 93(4), 747-751. doi: 10.4269/ajtmh.15-0300.

Dheda, K., Barry, C. E., \& Maartens, G., 2016. Tuberculosis. The Lancet, 387(10024), 12111226. doi: 10.1016/S0140-6736(15)00151-8.

Dhanaraj, B. Papanna, M. K., Adinarayanan, S., Vedachalam, C., Sundaram, V., Shanmugam, S., \& Swaminathan, S., 2015. Prevalence and risk factors for adult pulmonary tuberculosis in a metropolitan city of south India. PLoS one, 10(4). Availabe from: https://www.ncbi.nlm.nih.gov/pmc/articles/P MC4408069/pdf/pone.0124260.pdf. Access: Aug 282019.

Dos Santos, D. T., Alves, L. S., Arcoverde, M. A. M., Arroyo, L. H., Berra, T. Z., Ramos, A. C. V., Lima dos Santos, F., Arcêncio, R. A., \& Nunes, C., 2020. Social risk and its association with tuberculosis mortality in a context of high inequality in South Brazil: A geo-epidemiology analysis. Journal of Infection and Public Health, 1-8. doi: 10.1016/j.jiph.2020.03.010.

Duarte R, Lönnroth K, Carvalho C, Lima F, Carvalho A. C. C., Muñoz-Torrico M., \& Centis, R., 2018. Tuberculosis, social determinants and co-morbidities (including HIV). Pulmonology 24(2), 115-119.

Garcia, L. P., Silva G. D. M., 2016. Doenças transmissíveis e situação econômica no Brasil: análise espacial. Brasília-DF: Ipea.

Gehlen, M., Nicola, M. R. C., Costa, E. R. D., Cabral, V. K., de Quadros, E. L. L., Chaves, C. O., Lahm, R. A., Nicolella, A. D. R., Rossetti, M. L. R., \& Silva, D. R., 2019. Geospatial intelligence and health analitycs: Its application and utility in a city with high tuberculosis incidence in Brazil. Journal of Infection and Public Health. 12, 681-689. doi.org/10.1016/j.jiph.2019.03.012.

Gelaw, Y. A., Yu, W., Magalhães, R. J. S., Assefa, Y., \& Williams, G., 2019. Effect of temperature and altitude difference on tuberculosis notification: a systematic review. Journal of global infectious diseases, 11(2),
63-68. doi: 0.4103/jgid.jgid_95_18: 10.4103/jgid.jgid_95_18.

Geodacenter., $2020 . \quad$ Disponível: https://geodacenter.github.io/download_wind ows.html. Acesso: 28 jun. 2020.

Guimarães, R. B., 2016. Geografia e saúde coletiva no Brasil. Saúde e sociedade, 25, 869-879. doi: 10.1590/S010412902016167769 .

Hosmer, D. W., Lemeshow, S., \& Sturdivant, R, X., 2013. Applied Logistic Regression. Wiley Series in Probability and Statistics. New York: John Wiley \& Sons. Availabe: http://search.lib.virginia.edu/catalog/ocn8301 63779. Access: Sep 282017.

Izumi, K., Ohkado. A., Uchimura, K., Murase, Y., 2015. Detection of tuberculosis infection hotspots using activity spaces based spatial approach in an urban Tokyo, from 2003 to 2011. PLoS One. 416,9,1-16. Availabe: http://dx.doi:10.1371/journal.pone.0138831. Access: Jun 202020.

Jerrett, M., Gale, S., \& Kontgis, C., 2010. Spatial modeling in environmental and public health research. International journal of environmental research and public health, 7 , 1302-1329. doi: 10.3390/ijerph7041302.

Júnior, A. N., 2016. Mortality related to tuberculosis-HIV/AIDS co-infection in Brazil, 2000-2011: epidemiological patterns and time trends. Cadernos de saúde pública (Online), 32(10), e00026715-e00026715. Retrieved from

http://www.scielo.br/scielo.php?script=sci ar ttext\&pid=S0102-311X2016001005008.

Kolifarhood, G., Khorasani-Zavareh, D., Salarilak, S., Shoghli, A., \& Khosravi, N., 2015. Spatial and non-spatial determinants of successful tuberculosis treatment outcomes: An implication of Geographical Information Systems in health policy-making in a developing country. Journal of epidemiology and global health, 5(3), 221-230. doi: 10.1016/j.jegh.2014.11.001.

Leal, B. D. N., Mesquita, C. R., Nogueira, L. M. V., Rodrigues, I. L. A., Oliveira, L. F. de, \& Caldas, R. J. C., 2019. Análise espacial em 
tuberculose e a rede de atenção primária em saúde. Revista Brasileira de Enfermagem, 72(5), 1262-1267.

Lee, E. C., Asher, J. M., Goldlust, S., Kraemer, J. D., Lawson, A. B., \& Bansal, S., 2016. Mind the scales: Harnessing spatial big data for infectious disease surveillance and inference. The Journal of infectious diseases, 214(Suppl 4), S409-S413. doi: 10.1093/infdis/jiw344.

Liao, W. Bin, Ju, K., Gao, Y. M., \& Pan, J., 2020. The association between internal migration and pulmonary tuberculosis in China, 20052015: a spatial analysis. Infectious Diseases of Poverty, 9(1), 1-12. doi: 10.1186/s40249-0200621-x.

Lima, M. D. S., Martins-Melo, F. R., Heukelbach, J., Alencar, C. H., Boigny, R. N., \& Ramos Júnior, A. N., 2016. Mortality related to tuberculosis-HIV/AIDS co-infection in Brazil, 2000-2011: epidemiological patterns and time trends. Cadernos de saúde pública (Online), 32(10), e00026715-e00026715. Retrieved from

http://www.scielo.br/scielo.php?script=sci ar ttext\&pid=S0102-311X2016001005008.

Liu, F., Zhang, Z., Chen, H., \& Nie, S., 2020. Associations of ambient air pollutants with regional pulmonary tuberculosis incidence in the central Chinese province of Hubei: A Bayesian spatial-temporal analysis. Environmental Health: A Global Access Science Source, 19(1), 1-10. doi: 10.1186/s12940-020-00604-y.

Luzardo, A. J. R., Castañeda Filho, R. M., \& Rubim, I. B., 2017. Análise espacial exploratória com o emprego do Índice de Moran. Geographia, 19(40), 161-179.

Magalhães, M. D. A. F. M., \& Medronho, R.A., 2017. Análise espacial da tuberculose no Rio de Janeiro no período de 2005 a 2008 e fatores socioeconômicos associados utilizando microdado e modelos de regressão espaciais globais. Ciência \& Saúde Coletiva, 22, 3:831840. Disponível: http://dx.doi:10.1590/141381232017223.24132015. Acesso: 20 jun. 2020.
McClelland G. H, Irwin J. R, Disatnik D, \& Sivan L., 2016. Multicollinearity is a red herring in the search for moderator variables: A guide to interpreting moderated multiple regression models and a critique of Iacobucci, Schneider, Popovich, and Bakamitsos. Behavior research methods. 49(1), 394-402.

Ministério da Saúde., 2018. Informações de Saúde: DATASUS [online]. Disponível: http://tabnet.datasus.gov.br/cgi/tabcgi.exe?sin annet/cnv/tubercsc.def. Acesso: 22 fev. 2019.

Ministério da Saúde., 2017. Apresentação dos principais indicadores da tuberculose [online]. Disponível:http://portalarquivos2.saude.gov.b r/images/pdf/2018/julho/11/APRES-

PADRAO-MAI-18-periodo-eleitoralreduzida.pdf. Published 2017. Acesso: 28 set. 2017.

Ministério da Saúde (SVS)., 2017. Brasil livre da tuberculose: plano nacional pelo fim da tuberculose como problema de saúde pública. Brasília-DF: Ministério da Saúde. Disponível: http://bvsms.saude.gov.br/bvs/publicacoes/br asil_livre_tuberculose_plano_nacional.pdf. Acesso: 28 set. 2017.

Ministério da Saúde. Secretaria de Vigilância em Saúde., 2016. Série histórica da taxa de incidência de tuberculose. Brasil, Regiões e Unidades Federadas de residência por ano de diagnóstico (1990 a 2015) [online]. Disponível:

http://portalarquivos.saude.gov.br/images/pdf /2017/janeiro/03/taxa-incidencia-tuberculose1990-2015-OUTUBRO-2016.pdf. Acesso: 28 set. 2017.

Ministério da Saúde., 2016. E-Gestor atenção básica: informação e gestão de antenção básica [online]. Disponível: https://egestorab.saude.gov.br/paginas/acesso Publico/relatorios/relHistoricoCoberturaAB.x html. Acesso: 22 fev. 2017.

Ministério do Planejamento (IBGE)., 2012. Nupcialidade, fecundidade e migração. Censo demográfico 2010: resultado da mostra. Brasília: IBGE. Disponível em: https://biblioteca.ibge.gov.br/visualizacao/per iodicos/98/cd_2010_nupcialidade_fecundida de_migracao_amostra.pdf. Acesso: 28 set. 2017. 
Ministério do Planejamento Orçamento e Gestão (IBGE)., 2011. Aglomerados subnormais. Informações territoriais. Censo demográfico 2010. Brasília: IBGE. Disponível: https://biblioteca.ibge.gov.br/visualizacao/per iodicos/552/cd_2010_agsn_if.pdf. Acesso: 28 set. 2017.

Ministério do Planejamento Orçamento e Gestão (IBGE), 2018. População [online]. Disponível:

https://www.ibge.gov.br/estatisticas/sociais/p opulacao.html. Acesso: 20 jan. 2019.

Ortblad K. F., Salomon, J. A., Barninghausen T, Atun R., 2015. Stopping tuberculosis: a biosocial model for sustainable development. The Lancet, 386 (10010), 2354-2362.

Pareek M, Greenaway C., Noori T, Munoz, J., \& Zenner, D., 2016. The impact of migration on tuberculosis epidemiology and control in high-income countries: a review. BMC medicine [online]. 14(1), 48. Available: http://dx.doi.org/10.1186/s12916-016-0595-

5. Access: Jun 292020.

Pelissari, D. M., \& Diaz-Quijano, F. A., 2017. Household crowding as a potential mediator of socioeconomic determinants of tuberculosis incidence in Brazil. PLoS One. Availabe: http://dx.doi:10.1371/journal.pone.0176116. Access: Jun 202020.

Prasad, A., Ross, A., Rosenberg, P., \& Dye, C., 2016. A world of cities and the end of TB. Transactions of The Royal Society of Tropical Medicine and Hygiene, 110(3),151-152. Availabe: http://dx.doi:10.1093/trstmh/trw004. Access: Jun 202020.

Programa das Nações Unidas para o Desenvolvimento, Instituto de Pesquisa Econômica Aplicada FJP., 2012. Atlas Brasil: Atlas do Desenvolvimento Humano no Brasil [online].

Disponível: http://atlasbrasil.org.br/2013/pt/destaques/fai xas_idhm/. Acesso: 23 jun. 2017.

Qgis., 2020. Um sistema de informação geográfica livre e aberto. Disponível: https://www.qgis.org/pt_BR/site/. Acesso em 28 jun. 2020.
Rao, H. X., et al., 2016. Spatial transmission and meteorological determinants of tuberculosis incidence in Qinghai Province, China: a spatial clustering panel analysis. Infectious diseases of poverty, 5, 45. Availabe: https://idpjournal.biomedcentral.com/track/pd f/10.1186/s40249-016-0139-4. Access: Aug 282019.

Reichler M. R., Khan, A., Sterling, T. R., Zhao, H., Moran, J., McAuley, J., et al., 2018. Risk and timing of tuberculosis among close contacts of persons with infectious tuberculosis. The Journal of infectious diseases, 218(6), 1000-1008.

Righetto, A. J., \& Tachibana, V. M., 2015. Análise de regressão linear: abordagem tradicional e espacial em um estudo de caso. Revista Brasileira de Qualidade de Vida, 7, 3, 169-179. Disponível: https://periodicos.utfpr.edu.br/rbqv/article/vie w/3026/2210. Acesso: 25 ago. 2019.

Rodrigues, N. C. P., Andrade, M. K. N., O’Dwyer, G., Flynn, M., Braga, J. U., Almeida, A. S. D., et al., 2017. Distribution of pulmonary tuberculosis in Rio de Janeiro (Brazil): a spatial analysis. Ciência \& Saúde Coletiva, 22(12), 4125-4134. Availabe: http://dx.doi:10.1590/1413-

812320172212.0143016. Access: Jun 20 2020.

Sales, C. M. M., Nunes, G. F., Rogério, W., Castro, T., Santos, B. R., \& Maciel, E. L. N., 2015. Tuberculose e a questão social: uma revisão sistemática de estudos brasileiros. Revista Brasileira de Pesquisa, 17(4), 156175.

Sánchez, A., \& Larouzé, B., 2016. Controle da tuberculose nas prisões, da pesquisa à ação: a experiência do Rio de Janeiro, Brasil. Ciência \& Saúde Coletiva, 21(7), 2071-2080. Disponível: http://dx.doi:10.1590/141381232015217.08182016. Acesso: 20 jun. 2020.

Pedro, A. S., Gibson, G., Santos, J. P. C., Toledo, L. M. D., Sarboza, P. C., \& de Oliveira, R. M., 2017. Tuberculose como marcador de iniquidades em um contexto de transformação 
socioespacial. Revista de Saúde Pública, 5l(9), 1-10.

Schootman, M., Nelson, E. J., Werner, K., Shacham, E., Elliott, M., Ratnapradipa, K., Lian, M., \& McVay, A., 2016. Emerging technologies to measure neighborhood conditions in public health: implications for interventions and next steps. International journal of health geographics, 15(1), 1-9. doi: 10.1186/s12942-016-0050-z.

Secretaria do Estado da Fazenda. Caderno de Indicadores., 2016. Santa Catarina e suas regiões. Índice de Competitividade RegionalICR-SC. Diretoria de Planejamento Orçamentário. Diponível: http://www.sef.sc.gov.br/arquivos_portal/rela torios/11/Caderno_de_indicadores_2016

Revisao_final_junho_0.pdf. Acesso: 28 set. 2017.

Secretaria Estadual de Saúde de Santa Catarina (DIVE)., 2017. Situação da tuberculose em Santa Catarina 2001-2015. Informativo Epidemiológico Barriga Verde [online]. Disponível:

http://dive.sc.gov.br/conteudos/publicacoes/I nformativo_Tuberculose-2017.pdf. Acesso: 28 set. 2017.

Silva I. V., Campos, R. B., Antonio R, Oscar S., 2019. Tuberculose em Áreas de Fronteira: Óbitos e Doenças Associadas. Revista Pleiade, 12(26), 14-22.

Simpson, P. L., Simpson, M., Adily, A., Grant, L., \& Butler, T., 2019. Prison cell spatial density and infectious and communicable diseases: A systematic review. BMJ open, 9(7), 1-10. doi: 10.1136/bmjopen-2018026806.

Smith, C. M., Maguire, H., Anderson, C., Macdonald, N., \& Hayward, A. C., 2017. Multiple large clusters of tuberculosis in London: a cross-sectional analysis of molecular and spatial data. ERJ Open Research, 3(1), 1-12. doi: 10.1183/23120541.00098-2016.
Teixeira De Siqueira-Filha N, Militao De Albuquerque MDF, Cunha Rodrigues L, Legood R, Costa Santos A., 2018. Economic burden of HIV and TB/HIV coinfection in a middle-income couTrenntry: A costing analysis alongside a pragmatic clinical trial in Brazil. Sex Transm Infect. 94(6):463-9.

World Health Organization., 2015. Global tuberculosis report 2015. World Health Organization. Availabe: https://apps.who.int/iris/bitstream/handle/106 65/191102/9789241565059_eng.pdf?sequenc e=1\&isAllowed=y. Access: Aug 182019.

World Health Organization., 2016. Global tuberculosis report 2016. World Health Organization. Availabe: https://apps.who.int/medicinedocs/documents /s23098en/s23098en.pdf. Acesso: Aug 18 2019.

World Health Organization., 2017. Global tuberculosis report 2017. Availabe: https://www.who.int/tb/publications/global_r eport/gtbr2017_main_text.pdf. Acesso: Sep 282017.

World Health Organization., 2018. Global tuberculosis report 2018. Availabe: https://www.who.int/tb/publications/global_r eport/en/. Acesso: Sep 282017.

World Health Organization. Global tuberculosis report., 2019. WHO Library. Disponível: https://www.who.int/tb/publications/global_r eport/tb19_Exec_Sum_12Nov2019.pdf?ua=1 Acesso: Jan 232020.

Wubuli A, Xue, F., Jiang, D., Yao, X., Upur, H., \& Wushouer, Q., 2015. Socio-demographic predictors and distribution of pulmonary tuberculosis (TB) in Xinjiang, China: A spatial analysis. PLoS One. 10(12), 1-22.

Zhang X., 2017. Spatial-temporal epidemiological characteristics of tuberculosis in Shandong Province, China in 2011-2015. EC Pulmonol. Respir. Med. [online]. Availabe: https://doi.org/10.1590/15188345.1064.2888. Access: Jun 282020. 